\title{
HIV-1 Infection, Injecting Drug Use, and Neuroendocrine Response to Psychological Stress
}

\section{Raymond L Ownby,*, Drenna Waldrop-Valverde², Adarsh M Kumar ${ }^{3}$, Deborah Jones ${ }^{3}$, Benny Fernandez ${ }^{3}$, and Mahendra Kumar $^{3}$}

${ }^{1}$ Department of Psychiatry and Behavioral Medicine, Nova Southeastern University, USA

${ }^{2}$ Nell Hodgson Woodruff College of Medicine, Emory University, USA

${ }^{3}$ Department of Psychiatry and Behavioral Sciences, University of Miami School of Medicine, USA

*Corresponding author: Raymond L Ownby, MD, PhD, Department of Psychiatry and Behavioral Medicine, Nova Southeastern University, Center for Collaborative Research, 3301 College Ave, Fort Lauderdale FL, USA. Telephone: +1-954-262-1481; E-mail: ro71@nova.edu

Received: 31 Jul, 2019 | Accepted: 25 Sep, 2019 | Published: 30 Sep, 2019

Citation: Ownby RL, Waldrop-Valverde D, Kumar AM, Jones D, Fernandez B, et al. (2019) HIV-1 Infection, Injecting Drug Use, and Neuroendocrine Response to Psychological Stress. J HIV AIDS 5(2): dx.doi.org/10.16966/2380-5536.167

Copyright: (C) 2019 Ownby RL, et al. This is an open-access article distributed under the terms of the Creative Commons Attribution License, which permits unrestricted use, distribution, and reproduction in any medium, provided the original author and source are credited.

\begin{abstract}
Background: Previous studies have suggested that HIV-1 infection is associated with neuroendocrine abnormalities including alterations in autonomic nervous system (ANS) activity. The norepinephrine (NE) response to cold pressor stress, an $\alpha$-adrenergic challenge, is blunted in HIV-1 infection. Given the relation of ANS activity to the function of the hypothalamic-pituitary-adrenal (HPA) axis and its role in cognitive functioning, changes in response to stress may be a factor in HIV-related cognitive dysfunction.
\end{abstract}

Objective: In this study, we evaluated the NE and cortisol response of persons in three groups:

Design/Participants: We studied stress response in three groups: (1) those with HIV-1 infection and a history of injecting drug use (IDU), those with HIV-1 infection but no IDU, and a control group of uninfected individuals without a history of IDU. Stress was induced by administering a neuropsychological test known to induce an immediate increase in NE, the Stroop Color-Word Test. Blood samples were obtained immediately before and after participants completed the Stroop and then at two intervals over the next 20 minutes. Data were analyzed using mixed-effects repeated measures models.

Main Measures: Serum norepinephrine, epinephrine, and cortisol.

Results: Analyses showed that those with both HIV-1 infection and history of IDU had a significantly greater NE response to stress that did not return to baseline over 20 minutes compared to those without infection or IDU history. Epinephrine and Cortisol responses followed similar patterns, but between-group differences were not statistically significant.

Conclusions: The combination of history of IDU and HIV infection may produce an exaggerated neuroendocrine response that does not quickly return to baseline levels. Given the potential impact of these on cognitive and physical function in affected these individuals, implementing stress management techniques with them may be important.

Keywords: HIV; Stress; Norepinephrine; Cortisol; Neuroendocrine response

\section{Introduction}

Infection with human immunodeficiency virus, type 1 (HIV-1) is associated with a variety of clinical manifestations. Neurological complications are often especially troublesome for affected individuals and may include memory and other cognitive deficits as well as impairments in the function of the autonomic nervous system or ANS [1]. Several studies have shown that individuals with HIV-1 infection may have multiple disturbances in their response to stress, suggesting that infection is associated with disturbances in autonomic reactivity $[1,2]$ that have implications for the effectiveness of treatment [3] as well as mood and sleep [4,5] and cognition [6].
Stress-induced change in neuroendocrine markers is a widely-used index of ANS function [7,8]. In an earlier study [2], we used the cold pressor test to study changes in plasma catecholamines in persons in the early stages of HIV infection. In this test, persons are asked to place one of their hands in an ice water bath for 1 to 2 minutes. Levels of plasma catecholamines are then studied over the next 5 to 10 minutes. This study suggested that persons in the early stages of HIV infection showed a blunted response to cold pressor stress, with lower norepinephrine levels at all time points after the stressor. In addition, results showed that a larger proportion of HIV+ individuals attained their peak norepinephrine response in the first time period 
after the stressor compared with HIV- individuals. These findings were interpreted as indicating a dysfunction of the autonomic nervous system in individuals with HIV infection. The precise relevance of results from this type of stressor, which involves an unpleasant physical experience, for other forms of stress, is unclear since this form of stress is rarely experienced in daily life.

Little is known about the catecholamine response to psychological stress in persons with HIV-1 infection. The potential of importance of knowing more about these persons' reaction to stress is great. Psychological stress among HIV+ individuals has been related to markers of immunological status and clinical outcomes. Understanding the autonomic response to stress in HIV+ individuals might contribute to a greater understanding of this relation.

Experimental methods for inducing stress include a variety of procedures, each with advantages and risks. Even standard neuropsychological measures have been shown to induce changes in levels of catecholamines in persons being evaluated [9]. One simple and brief measure that induces stress that can be detected by changes in neuroendocrine function is the Stroop Color-Word Test [10]. Persons asked to do this task typically experience this task as requiring conscious effort, and it has been shown to be sensitive to various forms of neuropsychological dysfunction including the effects of HIV-1 infection [11]. In a previous study, the Stroop task was shown to induce changes in catecholamine levels in persons asked to complete it [12].

Psychological stress of the sort induced by situations that require conscious subjective effort and may be mildly anxiety-provoking is likely to be a common experience among those infected with HIV. This type of stress may thus be particularly relevant to understanding the role of daily stress to immunological function in affected individuals. A greater understanding of the ways that this sort of stress affects catecholamine levels might thus be useful in understanding how it affects the lives of HIV+ individuals. The purpose of this study was thus to investigate autonomic nervous system reactivity in persons infected with HIV-1 through assessing differences between infected and non-infected persons in norepinephrine response to a psychological stressor the Stroop Color-Word Test.

\section{Method}

\section{Participants}

Community residing men and women, who were enrolled as part of a larger study of endocrine responses to stress in persons with HIV-1 infection and/or a history of injecting drug use (IDU), were included in this study. Participants for the present study included HIV positive and HIV negative individuals with a history of injecting drug use as well as control participants. None of the HIV-1 infected participants had been treated or was currently treated with antiretroviral therapy. Please note that this was the result of the time during which these data were collected (1999-2004). Guidelines at the time were that antiretroviral medications would not be started until a person's CD4 count was less than 400 . Participants were within the age range of 18 to 50 and potential participants were excluded if they reported a history of head injury with loss of consciousness ( $>30$ minutes), or a history of major psychiatric illness such as schizophrenia or bipolar disorder, hypertension or diabetes mellitus.

Participants were considered injecting drug users if they had a history of regular injecting drug use defined as weekly drug injection for a period of at least 6 months. Additional use of other illicit substances via any route was not an exclusion criterion for IDUs.
Information on the duration and time since last use of heroin, cocaine/ crack and marijuana was obtained from all participants. Eligible control participants did not meet diagnostic criteria for dependence on any substance, including alcohol, in at least two years. Substance use data was gathered using the Structured Clinical Interview for DSMIV diagnoses, or SCID [13]. All participants were required to have abstained from drugs and/or alcohol for at least 12 hours prior to each session. This was verified by self-report and urine toxicology screens. If a participant was found to be acutely intoxicated, the appointment was rescheduled for another time. HIV-positive participants were required to present evidence of their serostatus. Additionally, their plasma viral load was determined using PCR amplicor method (Roche Diagnostics; the Clinical Immunology Laboratory in the Department of Medicine, the University of Miami, School of Medicine). Verification of HIV seronegative status was not available as part of this study. HIV-positive participants included in the study were free of any AIDS-defining clinical symptoms. This study was approved by the University of Miami Institutional Review Board. All participants gave informed consent prior to initiation of the study and were paid for their participation.

\section{Psychological (Stroop) challenge}

Norepinephrine, epinephrine, and cortisol levels in 88 persons were assessed after administration of the Stroop Color Word Test [14], completed between 8:00 am and 11:00 am. On arrival, an indwelling venous catheter for drawing blood was placed in the antecubital vein of each participant. After 20-minutes of rest in a reclined position, a $9 \mathrm{ml}$ sample of blood was drawn in a tube containing EDTA to determine the resting, baseline level (T1) of norepinephrine, epinephrine, and cortisol (NE). The Stroop Color Word Test was then administered according to the Trenerry MR, et al. [14] method. In this version of the task, subjects are first given an $8 \times 10$ inch sheet of paper with the words blue, green, red and tan printed in colors that are consistent with the word (e.g., "red" is printed in red ink). One hundred words appear on each page and subjects are asked to read the words aloud as quickly as possible. In the second part of the Stroop task, subjects are again given an $8 \times 10$ inch sheet of paper with the color words, but in this instance the color of the ink is inconsistent with the word (e.g., "red" is written in blue). The subjects are then asked to rapidly state the color in which the words are printed. This condition requires that the participant inhibit the automatic response of read the word while considering the color of the ink. It is typically experienced as requiring conscious mental effort. Again, 100 words appear on each page. The test is scored as the number correct.

Immediately after completing the Stroop, a second blood sample of blood was drawn (T2). Two additional blood samples were collected at 10 minutes after completion of the Stroop (T3) and again 20 minutes after completion of the Stroop (T4). Samples of blood were immediately centrifuged at $4^{\circ} \mathrm{C}$ and plasma obtained was aliquoted and stored at $-80^{\circ} \mathrm{C}$ until assayed.

\section{Norepinephrine and epinephrine quantifications}

Norepinephrine (NE) and epinephrine (EPI) were assayed as described previously Kumar $\mathrm{M}$, et al., [2]. Briefly, to $1.0 \mathrm{ml}$ plasma, an internal standard, dihydroxybezylamine (DHBA) $500 \mathrm{pg} / 50 \mu \mathrm{l}$ was added and the mixture was treated with activated alumina $(\sim 20 \mathrm{mg})$ in the presence of $2 \mathrm{M}$ Tris buffer, $\mathrm{pH} 8.7$, mixed on a Roto shaker for 15 minutes at room temperature and was centrifuged (4500xg for 2 $\mathrm{min}$ ). The supernatant was discarded and after two cycles of washing with $0.2 \mathrm{M}$ tris, $\mathrm{pH} 8.7$, norepinephrine was eluted from alumina in $125 \mu \mathrm{l}$ of $0.1 \mathrm{M}$ glacial acetic acid containing $0.05 \%$ EDTA and $0.05 \%$ sodium metabisulphite. NE was assayed using a reverse phase HPLC 


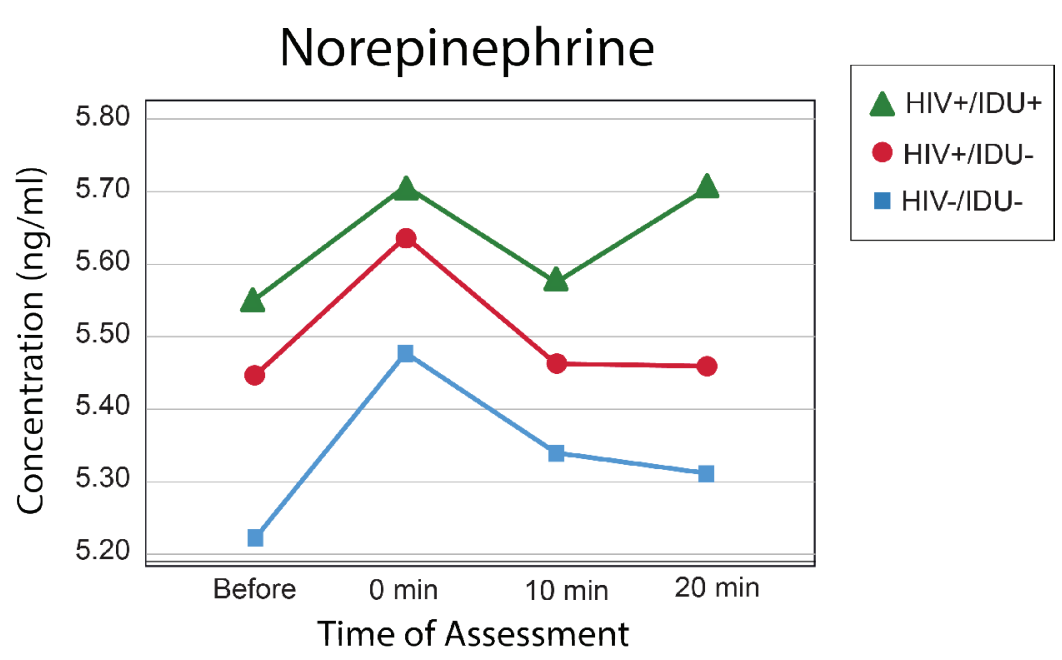

Figure 1: Norepinephrine levels.

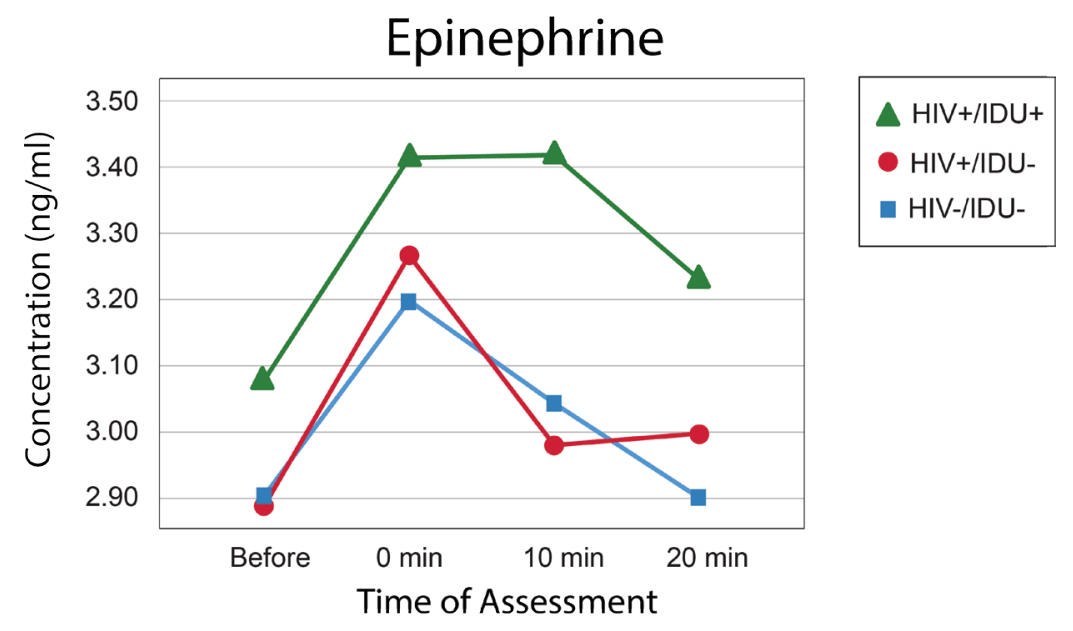

Figure 2: Epinephrine levels.

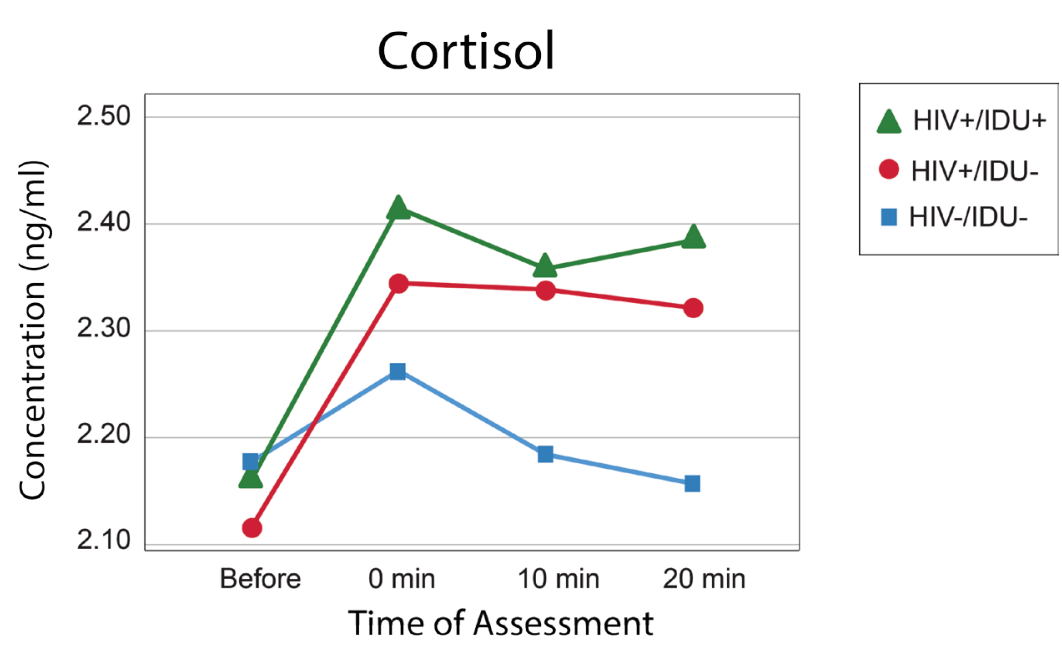

Figure 3: Cortisol levels. 
Table 1: Descriptive statistics for the sample.

\begin{tabular}{|c|c|c|c|c|c|c|c|c|c|}
\hline \multirow[b]{2}{*}{ Mean (SD) } & \multicolumn{2}{|c|}{$\begin{array}{c}\text { HIV-/IDU- } \\
(n=78)\end{array}$} & \multicolumn{2}{|c|}{$\begin{array}{c}\text { HIV-/IDU+ } \\
(n=19)\end{array}$} & \multicolumn{2}{|c|}{$\begin{array}{c}\text { HIV+/IDU+ } \\
(n=60)\end{array}$} & \multicolumn{2}{|c|}{$\begin{array}{c}\text { All } \\
(n=157)\end{array}$} & \multirow[t]{2}{*}{ Tests of Between-Group Differences } \\
\hline & Mean & SD & Mean & SD & Mean & SD & Mean & SD & \\
\hline Baseline In NE & 5.22 & 0.76 & 5.45 & 0.68 & 5.55 & 0.90 & 5.38 & 0.82 & $F(2,170)=2.82, p=0.06$ \\
\hline Baseline In Epi & 2.90 & 1.13 & 2.89 & 1.17 & 3.08 & 1.10 & 2.97 & 1.12 & $F(2,134)=0.68, p=0.51$ \\
\hline Baseline In Cort & 2.18 & 0.52 & 2.12 & 0.55 & 1.16 & 0.43 & 2.17 & 0.49 & $F(2,155)=0.06, p=0.94$ \\
\hline Age & 31.17 & 9.13 & 40.78 & 7.35 & 39.00 & 6.41 & 35.33 & 8.97 & $F(2,133)=20.09, p<0.001$ \\
\hline Education & 12.92 & 1.99 & 11.50 & 1.50 & 12.21 & 1.96 & 12.49 & 1.97 & $F(2,165)=9.11, p<0.001$ \\
\hline BMI & 28.52 & 6.46 & 25.76 & 6.67 & 25.95 & 4.97 & 27.36 & 6.02 & $F(2,166)=6.31, p=0.002$ \\
\hline SCW Score & 103.40 & 12.13 & 82.22 & 28.46 & 89.37 & 18.23 & 95.58 & 18.39 & $F(2,166)=10.38, p<0.001$ \\
\hline \multicolumn{10}{|l|}{ Counts } \\
\hline Whites & 30 & & 12 & & 14 & & 56 & & \\
\hline Blacks & 48 & & 7 & & 46 & & 101 & & \\
\hline Men & 31 & & 15 & & 41 & & 87 & & \\
\hline Women & 47 & & 4 & & 19 & & 70 & & \\
\hline
\end{tabular}

Table 2: Mixed Effects Model for Norepinephrine.

\begin{tabular}{|c|c|c|c|c|c|}
\hline \multicolumn{6}{|c|}{ Fixed Effects } \\
\hline Parameter & Estimate & Std. Error & df & $t$ & $p$ \\
\hline Intercept & 5.70 & 0.08 & 38.11 & 68.29 & $<0.001$ \\
\hline HIV-/IDU- & -0.34 & 0.12 & 59.34 & -2.82 & 0.01 \\
\hline HIV-/IDU+ & -0.21 & 0.20 & 21.95 & -1.05 & 0.31 \\
\hline
\end{tabular}

Table 3: Mixed Effects Model for Norepinephrine Pairwise Comparisons.

\begin{tabular}{|c|c|c|c|c|c|}
\hline \multicolumn{6}{|c|}{ Pairwise Group Comparisons } \\
\hline \multicolumn{2}{|c|}{ Comparison } & \multirow{2}{*}{$\begin{array}{c}\text { Mean Difference } \\
-0.13\end{array}$} & \multirow{2}{*}{$\begin{array}{c}\text { Std. Error } \\
0.20\end{array}$} & \multirow{2}{*}{$\begin{array}{c}\text { df } \\
25.29\end{array}$} & \multirow{2}{*}{$\begin{array}{c}p \\
0.88\end{array}$} \\
\hline & HIV-/IDU+ & & & & \\
\hline HIV-/IDU- & HIV+/IDU+ & $-0.34^{*}$ & 0.12 & 59.34 & 0.02 \\
\hline \multirow{2}{*}{ HIV-/IDU+ } & HIV-/IDU- & 0.13 & 0.20 & 25.29 & 0.88 \\
\hline & HIV+/IDU+ & -0.21 & 0.20 & 21.95 & 0.66 \\
\hline \multirow{2}{*}{ HIV+/IDU+ } & HIV-/IDU- & $0.34^{*}$ & 0.12 & 59.34 & 0.02 \\
\hline & HIV-/IDU+ & 0.21 & 0.20 & 21.95 & 0.66 \\
\hline
\end{tabular}

*Significant terms

separation followed by electrochemical detection (Waters, Alliance Separation module). Forty microliters of elute from alumina was injected into Waters $5 \mu \mathrm{m}$ spherical $\mathrm{C} 18$ guard and resolve column and NE was separated using isocratic mobile phase $(10 \%$ organic and $90 \%$ polar) as described earlier [2] at a flow rate of $0.7 \mathrm{ml} / \mathrm{min}$. NE was detected with electrochemical detected at $+0.70 \mathrm{~V}$ and identified by its retention time signal output was defined by area under the curve that was proportional to NE concentration. The concentrations of NE and Epi were determined using ratio method using Waters Empower software and expressed as $\mathrm{pg} / \mathrm{ml}$.

\section{Cortisol quantification}

A solid-phase radioimmunoassay (RIA) was used to quantify plasma cortisol concentration using a commercially available kit (DSL, Webster, TX). Briefly, samples of plasma were defrosted, vortexed and $25 \mu \mathrm{l}$ of plasma was added directly to cortisol antibody coated tubes, followed by the addition of $500 \mu \mathrm{l}$ of cortisol $\left[\mathrm{I}^{125}\right]$ reagent and mixed. The mixture was incubated for 45 minutes in a water bath at $37^{\circ} \mathrm{C}$. The tubes were centrifuged, decanted and radioactivity was determined in an automatic gamma-counter (Perkin-Elmer, Model 1470). The minimum detection limit of this technique is $0.3 \mu \mathrm{g}$ of cortisol per $\mathrm{dL}$ of plasma. The intra- and interassay coefficients of variance are $5.3 \%$ and $11.5 \%$ respectively.

\section{Data analyses}

Prior to evaluation of group differences in norepinephrine response, the distributions of norepinephrine, epinephrine, and cortisol were assessed across time points. For each, evaluation suggested the need for log transformation which was done prior to further. The relation of potential confounding variables, such as age, education, body mass index, race, and gender to these variables was assessed via correlations, with those variables having a significant $(\mathrm{p}<0.10)$ relation to any of the dependent variables included as covariates.

We used a linear mixed effects repeated measures model in SPSS 25 (Armonk, NY: IBM Corp.) to assess between-group differences, with HIV infection and IDU statuses as fixed effects and time, time squared, gender, race, and age as random effects. Repeated and random effects were modeled as a heterogeneous autocorrelation structure, with fit of the model assessed as the smallest value of Akaike's Information 
Criterion (AIC). Fit of the final model was confirmed via inspection of the distribution of residuals, which was approximately normal.

\section{Results}

Descriptive statistics for the samples included in the study are presented in table 1. Between-group differences on relevant variables were assessed with univariate ANOVAs for continuous variables and the chi-square statistic for categorical variables. These variables were included in the mixed effects models for NE, Epi, and cortisol. Fixed effects for group membership and between group comparisons are presented in table 2. Even after controlling for baseline norepinephrine level and the other variables included in table 3 , individuals who were infected with HIV-1 had higher norepinephrine levels in response to psychological stress than uninfected individuals (Figure 1).

Models with the same effects and covariates were created for Epi and cortisol (Figures 2 and 3), but no effects were statistically significant, and the models are not presented here. The pattern of norepinephrine, epinephrine, and cortisol responses between groups is illustrated in figures 1-3.

\section{Discussion and Conclusion}

These results thus show that even after controlling for several potentially relevant variables, persons with HIV-1 infection elevated NE levels in response to the psychological stress induced by the Stroop Color Word test when compared to uninfected individuals. Although no significant effects of group were obtained, the same pattern of response, with prolonged elevation at 20 minutes, occurred for epinephrine and cortisol. Results are thus consistent with differences in autonomic nervous system reactivity between those with and without HIV-1 infection as well as other studies showing that psychological stress is linked to activation of the HPA axis and the function of the sympathetic nervous system [15].

Limitations of this study include the significant between-groups differences on variables that could plausibly be important in participants' response to the psychological stressor. These include gender, age, education, and performance on the Stroop itself. Given the group differences in educational attainment and performance on the Stroop, it is possible that the observed differences in stress response over groups were the result of broader differences in general cognitive ability that might have an impact on coping. While these potential confounding variables are included as covariates in the analytic model, it is possible that this strategy may not fully account for the variables' impact on participants' neuroendocrine response to stress.

The practical significance of these findings is in their suggestion that individuals with HIV infection and injective drug use may have an exaggerated response to psychological stress. Elevated levels of cortisol have been associated with worse cognitive function in the general population $[16,17]$. Stress and elevated cortisol may also be related to the complex of chronic inflammation and other chronic diseases including diabetes, cardiovascular disease, and depression $[18,19]$. Given the effectiveness of stress management interventions in reducing cortisol levels [20,21], these findings emphasize the importance of providing persons with HIV better tools for managing stress.

\section{Support}

This study was supported by grants R01DA031201 and R01DA012792 to Dr. Kumar.

\section{Conflicts of Interest}

All authors have declared that they have no conflicts of interest.

\section{References}

1. Kumar M, Kumar AM, Waldrop $D$, Antoni $M H$, Schneiderman $N$, et al. (2002) The HPA axis in HIV-1 infection. J Acquir Immune Defic Syndr 31: S89-S93.

2. Kumar M, Morgan R, Szapocznik J, Eisdorfer C (1991) Norepinephrine response in early HIV infection. J Acquir Immune Defic Syndr 4: 782786.

3. Ironson G, Balbin E, Stieren E, Detz K, Fletcher MA, et al. (2008) Perceived stress and norepinephrine predict the effectiveness of response to protease inhibitors in HIV. Int J Behav Med 15: 221-226.

4. McIntosh R, Antoni M, Seay J, Fletcher MA, Ironson G, et al. (2019) Associations among Trajectories of Sleep Disturbance, Depressive Symptomology and 24-Hour Urinary Cortisol in HIV+ Women Following a Stress Management Intervention. Behav Sleep Med 17: 605-620.

5. McIntosh RC, Antoni M, Carrico A, Duran R, Hurwitz BE, et al. (2017) Change in urinary cortisol excretion mediates the effect of angry/ hostile mood on 9 month diastolic blood pressure in HIV+ adults. J Behav Med 40: 620-630.

6. Ownby RL, Waldrop-Valverde D, Kumar A, Mack A, Fernandez JB, et al. (2006) Cortisol Response mediates HIV-1 cognitive deficits among injecting drug abusers. Am J Infect Dis 2: 74-79.

7. King AP, Liberzon I (2009) Assessing the neuroendocrine stress response in the functional neuroimaging context. Neurolmage 47: 1116-1124.

8. Loucks EB, Robert JP, Jens PC (2008) Neuroendocrine biomarkers, allostatic load, and the challenge of measurement: A commentary on Gersten. Soc Sci Med 66: 525-530.

9. Folli D, Mutti A, Van der Venne MT, Berlin A, Gerra G, et al. (1992) Neuroendocrine response to psychological performance testing. Psychoneuroendocrinology 17: 467-474.

10. Stroop JR (1935) Studies of interference in serial verbal reactions. J Exp Psychol 18: 643-662.

11. Martin EM, Robertson LC, Edelstein HE, Jagust WJ, Sorensen DJ, et al. (1992) Performance of patients with early HIV-1 infection on the Stroop Task. J Clin Exp Neuropsychol 14: 857-868.

12. Tulen JH, Moleman P, van Steenis HG, Boomsma $F$ (1989) Characterization of stress reactions to the Stroop Color Word Test. Pharmacol Biochem Behav 32: 9-15.

13. First MB (1996) Structured Clinical Interview for DSM-IV Axis I Disorders, Patient Edition (SCID-I/P). Biometrics Research Department, New York State Psychiatric Institute, USA.

14. Trenerry MR, Crosson B, DeBoe J, Leber WR (1989) Stroop Neuropsychological Screening Test Manual. Odessa, Psychological Assessment Resources, Inc.

15. David S Goldstein (2003) Catecholamines and stress. Endocrine Regulations 37: 69-80.

16. Lee BK, Glass TA, McAtee MJ, Wand GS, Bandeen-Roche K, et al. (2007) Associations of salivary cortisol with cognitive function in the Baltimore Memory Study. Arch Gen Psychiatry 64: 810-818.

17. Lara VP, Caramelli P, Teixeira AL, Barbosa MT, Carmona KC, et al. (2013) High cortisol levels are associated with cognitive impairment no-dementia (CIND) and dementia. Clin Chim Acta 423: 18-22. 
18. Contoreggi C, Chrousos GP, Di Mascio M (2016) Chronic distress and the vulnerable host: A new target for HIV treatment and prevention? Neurobehav HIV Med 7: 53-75.

19. Valdez AN, Rubin LH, Neigh GN (2016) Untangling the Gordian knot of HIV, stress, and cognitive impairment. Neurobiol Stress 4: 44-54.

20. Jones D, Owens M, Kumar M, Cook R, Weiss SM (2014) The effect of relaxation interventions on cortisol levels in HIV-seropositive women. J Int Assoc Provid AIDS Care 13: 318-323.

21. Antoni $M H$, Cruess $S$, Cruess DG, Kumar M, Lutgendorf $S$, et al. (2000) Cognitive-behavioral stress management reduces distress and 24-hour urinary free cortisol output among symptomatic HIVinfected gay men. Ann Behav Med 22: 29-37. 